\title{
Systematic effects of bubble volume on the surface tension measured by pendant bubble profiles
}

\author{
Shi-Yow Lin ${ }^{\text {a,* }}$, Wei-Jiunn Wang ${ }^{a}$, Lung-Wei Lin ${ }^{a}$, Li-Jen Chen ${ }^{b}$ \\ a Department of Chemical Engineering, National Taiwan Institute of Technology, Taipei, 106, Taiwan \\ ${ }^{\mathrm{b}}$ Department of Chemical Engineering, National Taiwan University. Taipei, 106, Taiwan
}

Received 11 October 1995; accepted 21 December 1995

\begin{abstract}
The surface tensions of five different liquids water, glycerol, ethylene glycol, benzaldehyde and toluene, are measured at $25^{\circ} \mathrm{C}$ by using the pendant bubble shape technique at different bubble volumes. It is found that the surface tension measured is a function of the bubble volume: the larger the volume, the more accurate the tension. This phenomenon is simply due to the accuracy limit on the edge detection of a bubble image. Three needles of different inner diameters are used to generate bubbles in the liquid phase. A needle with a larger diameter has a larger range of volumes for obtaining the correct surface tension within acceptable measurement error (less than 0.2 or $0.5 \mathrm{mN} \mathrm{m}^{-1}$ ). A universal relationship between surface tension, capillary constant and bubble volume is proposed for our system by using the dimensionless analysis method.
\end{abstract}

Keywords: Bubble volume; Capillary constant; Drop volume; Pendant drop method; Surface tension

\section{Introduction}

The video-enhanced pendant drop method is currently one of the most popular techniques for surface/interface tension measurements [1-7]. This technique is capable of measuring static and dynamic tensions and is applicable to the measurement of high and ultra-low interfacial tensions of fluid-fluid interfaces $[8,9]$.

Whereas the Young-Laplace equation is dropsize independent, Lin et al. [10] showed that drops of different sizes may exhibit different interfacial tensions and the error may approach 15\%. Drops or bubbles with smaller absolute values of the capillary constant show larger measurement errors due to the accuracy limit on the edge detection of drop images. The measurement error of interfacial

* Corresponding author. tension (IFT) on the drop volume was also examined by two different systems: one with a high surface tension (air-water interface; $\gamma=72 \mathrm{mN}$ $\mathrm{m}^{-1}$ ), and the other with a low IFT (liquid-liquid interface of the $n$-tetradecane/water/diethylene glycol monohexyl ether system; $\gamma=0.2 \mathrm{mN} \mathrm{m}^{-1}$ ).

The IFT for most fluid interfaces, and that which most investigators have measured, ranges from 20 to $70 \mathrm{mN} \mathrm{m}^{-1}$. The aim of this paper is to present the relationship of the measured surface tension to the bubble volume for different liquids with surface tensions within this range. The volume limitation of the pendant drop used to obtain the correct surface tension is also presented. These data will be helpful when estimating and predicting the measurement error when a pendant drop system is utilized for IFT measurements.

In section 2, we shall describe briefly the experimental apparatus, the edge detection routine, and 
the experimental protocol. Section 3 is devoted to the calculation of the surface tension and the maximum bubble volume available for a specific needle and liquid phase. Experimental data on the bubble volume dependence of the surface tension measured using a video-enhanced pendant bubble tensiometer is presented in section 4. Finally, a discussion on how to predict the experimental error when using different sizes of needle, a universal relationship between the measured surface tension and dimensionless parameters, and a conclusion are presented in section 5 .

\section{Apparatus, materials and methods}

Pendant drop tensiometry enhanced by video digitization is employed for the measurement of the surface tension of the air-liquid interface. The pendant drop apparatus has been described in detail in a previous study [10], and therefore only a brief description is given here.

Fig. 1 shows schematically the pendant drop tensiometry set-up. There are three major parts to this apparatus: (i) an image-forming and image- digitizing system; (ii) a bubble/drop-forming system; and (iii) a temperature-control system. The first part consists of a parallel light generator, a solid state CCD camera, a video image digitizer, a recorder, and a frame code generator. The drop image on the active area of the camera is magnified approximately 1.5 times. The image digitizer digitizes a picture into $480 \times 512$ pixels with 8 bit resolution of a gray level. The second part consists of a computer-controlled three-way solenoid valve, a syringe and syringe pump, and inverted stainless steel needles. Three needles of different diameters with a $90^{\circ}$ bevel (Hamilton) are used: 16 gauge (0.047 in i.d.; 0.065 in o.d.); 22 gauge ( 0.016 in i.d.; 0.028 in o.d.); 24 gauge ( 0.010 in i.d.; 0.022 in o.d.). The third part is an air thermostat which is made from an acrylic material and consists of a cooling copper coil and water circulator, a heater, a fan, and a humidifier. The temperature stability of the liquid in a quartz cell inside the air thermostat is better than $\pm 0.02^{\circ} \mathrm{C}$ for temperatures ranging from 10 to $30^{\circ} \mathrm{C}$.

An edge detection routine is devised for locating the interface contour from the digitized image. The edge is defined as the $x$ or $z$ position that corres-

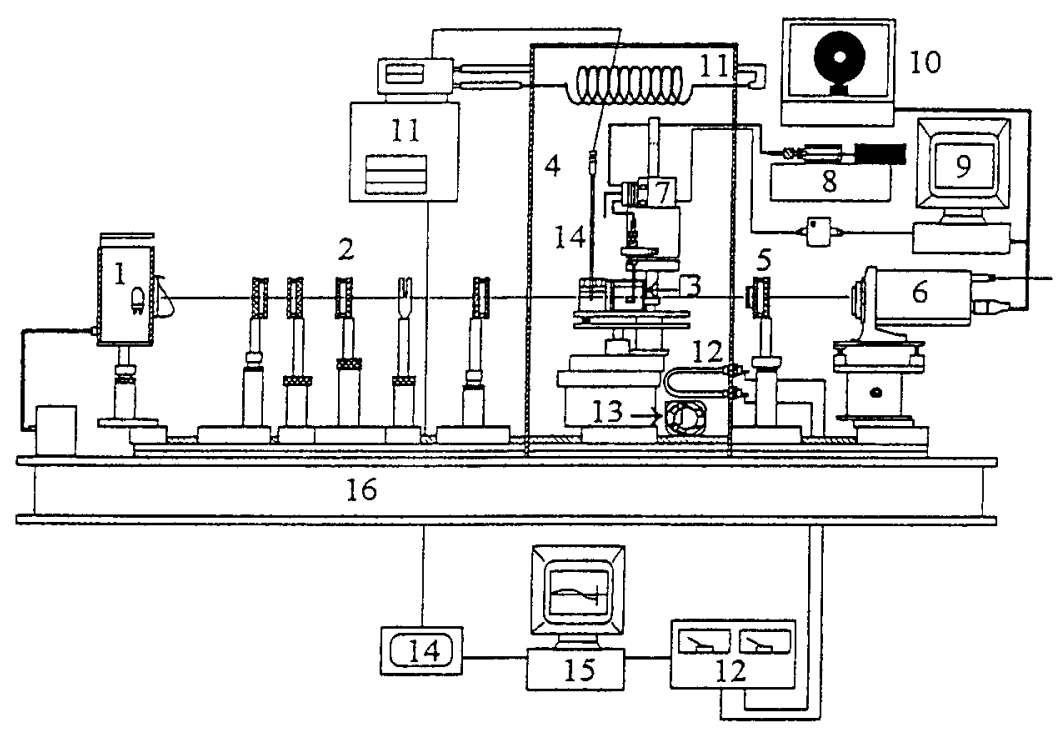

Fig. 1. Schematic illustration of the pendant drop tensiometry apparatus and the video digitization equipment: 1 , light source; 2 , lens, pin holes, and filters; 3, quartz sample cell and needle; 4, air thermostat; 5 , objective lens; 6 , CCD, video camera; 7, solenoid valve; 8, syringe and syringe pump; 9 , frame grabber, video recorder, D/A card, and personal computer; 10, monitor; 11, circulator and copper tube; 12, heater; 13 , fan; 14, thermometer; $15, \mathrm{D} / \mathrm{A}, \mathrm{A} / \mathrm{D}$ card and personal computer; 16 , vibration-isolation table. 
ponds to an intensity of 127.5. Two stainless steel balls $(1.577 \pm 0.002 \mathrm{~mm}$ and $1.983 \pm 0.002 \mathrm{~mm}$ in diameter, calibrated by a digital linear gauge) are used to calibrate the distance between pixels both horizontally and vertically. The calibration procedure yields values of 100.86 pixel $\mathrm{mm}^{-1}$ horizontally and 124.56 pixel $\mathrm{mm}^{-1}$ vertically.

Benzaldehyde $\left(\mathrm{C}_{6} \mathrm{H}_{5} \mathrm{CHO}\right.$, purity $\left.>99 \%\right)$, ethylene glycol $\left(\mathrm{HOCH}_{2} \mathrm{CH}_{2} \mathrm{OH}\right.$; purity $\left.>99.5 \%\right)$, and toluene $\left(\mathrm{C}_{6} \mathrm{H}_{5} \mathrm{CH}_{3}\right.$; purity $\left.>99.5 \%\right)$ were obtained from Ferak. Glycerol $\left(\mathrm{C}_{3} \mathrm{H}_{8} \mathrm{O}_{3}\right.$; purity $\left.>98 \%\right)$ was purchased from Merck. These chemicals are used as received without further purification. Water is purified via a Barnstead NANOpure II water purification system, with the output water having a specific conductance of less than $0.057 \mu \Omega^{-1} \mathrm{~cm}^{-1}$.

The experimental protocol was as follows. The quartz cell was initially filled with liquid, and the bubble-forming needle was positioned in the cell in the path of the collimated light beam. At this point, the solenoid valve was not energized. The syringe pump was turned on, and the air in the syringe was allowed to pass through the normally open port of the valve. Via an input from the keyboard, the D/A card energized the valve and allowed the gas to pass through the needle, thereby forming a bubble of air. The valve was then closed (also by an input from the keyboard) when the bubble achieved the correct diameter. The time required to create an air bubble was about $0.1 \mathrm{~s}$. Fig. 2 shows the change in the surface area of the

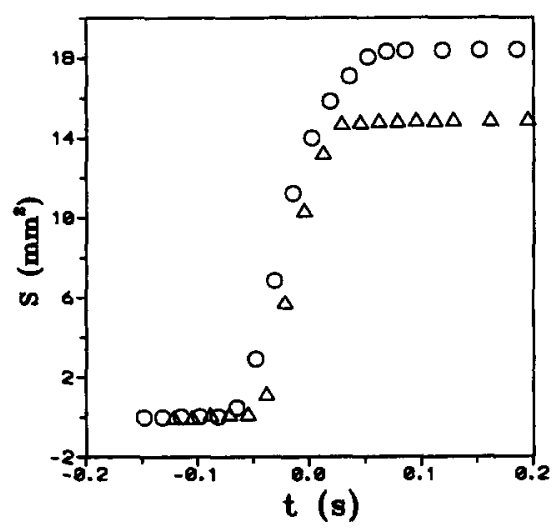

Fig. 2. Representative relaxation of the surface area on the formation of a pendant bubble on the tip of a 22 gauge needle. Each symbol represents a separate bubble. bubble during bubble formation. After the solenoid was closed and the bubble had formed, sequential digital images of the bubble were then taken. The images could also be recorded during this process and passed sequentially to the computer. The images were then processed in order to determine the bubble edge coordinates by a sub-pixel resolution procedure $[5,11]$, and the surface tension was determined by fitting with the Young-Laplace equation, the details of which are given in the following section. All the surface tensions of the air-liquid interface were measured at $25^{\circ} \mathrm{C}$.

\section{Calculation of surface tension and maximum volume of pendant bubble}

The classical Laplace equation relates the pressure difference across a curved interface [4] by

$\gamma\left(\frac{1}{R_{1}}+\frac{1}{R_{2}}\right)=\Delta P$

where $\gamma$ is the surface tension, $R_{1}$ and $R_{2}$ are the two principal radii of curvature of the interface, and $\boldsymbol{A P}$ is the pressure difference across the interface. Eq. (1) is recast as a set of three first-order differential equations in terms of the spatial positions $x$ and $z$ and the turning angle $\phi$ of the interface as a function of the arc length $s$. Eq. (1) is then integrated by using a fourth-order RungeKutta scheme [12] initialized with an approximate solution [3] which is valid near the apex.

The surface tension is computed from the data and the theoretical curve by adjusting four unknown parameters: the actual location of the apex $\left(X_{0}\right.$ and $\left.Z_{0}\right)$, the radius of curvature at the apex $\left(R_{0}\right)$, and the capillary constant $(B)$. The Newton-Raphson method [12] is applied to the minimization procedure in order to obtain optimum values of these four parameters. From the optimum values of $R_{0}$ and $B$, the tension $\gamma$ can be computed.

From the data sets, there are approximately 300-900 measured edge coordinates for one image of the drops or bubbles of different volumes. To obtain the minimum distance $\left(d_{n}\right)$ between the edge point and the theoretical bubble profile for each 
point, a theoretical curve consisting of 8000 points is first generated, and $d_{\mathrm{n}}$ is then computed as the distance from the experimental point to the closest of the discrete theoretical points. Initial guesses for the Newton-Raphson procedure are obtained in the following way: $X_{0}$ is initially guessed as the centroid of the 300-900 $\times$ location data points, and $Z_{0}$ as the average of the three highest $z$ coordinates. Initial guesses for $R_{0}$ and $B$ are obtained as follows. The equatorial diameter $\left(d_{\mathrm{e}}\right)$ and the diameter $\left(d_{s}\right)$ in a selected plane at a distance $d_{\mathrm{e}}$ from the apex of the bubble are obtained from the raw data. By integrating Eq. (1) for different values of $B$, graphs can be constructed of $B$ as a function of $d_{\mathrm{s}} / d_{\mathrm{e}}(=S)$ and $d_{\mathrm{e}} /\left(2 R_{0}\right)$ as a function of $B$. The best fit curves can be described by the following equations [10] $(-0.1>B>-0.58)$ :

$$
\begin{aligned}
B= & 0.688001 S^{3}-2.067220 S^{2}+0.951414 S \\
& -0.168520
\end{aligned}
$$

$$
\begin{aligned}
d_{\mathrm{e}} /\left(2 R_{0}\right)= & 0.484004 S^{3}-0.658246 S^{2}+0.427795 S \\
& +0.912193
\end{aligned}
$$

As $d_{\mathrm{s}}$ and $d_{\mathrm{e}}$ are known from the raw data, $S, B$, $R_{0}$ and therefore $\gamma$ can be obtained. Usually, 2-5 iterations are necessary for the program to converge, and the standard deviation of the distance between a measured point and the calculated curve is about 0.1 pixel (approximately $1 \mu \mathrm{m}$ ).

The maximum volume $\left(V_{\max }\right)$ of a pendant bubble is calculated from the theoretical curve in the following way. Eq. (1) is integrated to obtain a theoretical bubble profile by selecting $B$ $\left(=\Delta \rho g R_{0}^{2} / \gamma\right.$, capillary constant) and $R_{0}$ (radius of curvature at the apex). Once a liquid is chosen for the measurement, $\Delta \rho$ and $\gamma$ are fixed. There is only one unknown parameter, i.e. $R_{0}$, left. For a pendant bubble with the maximum volume for a selected needle and liquid system, the neck of the bubble is just attached to the tip of the needle. In other words, the diameter of the neck of the bubble with the maximum volume is equal to the inner diameter of the needle. Therefore the drop profile and its maximum volume are uniquely determined once the size of the needle and the liquid phase are selected. During the calculation, a trial and error estimation of the $B$ value is carried out in order to find the correct diameter of the bubble neck that is the same as that of the needle used.

\section{Results}

Consider first the air-water system. Air bubbles with different volumes are created on the tips of converted needles of 16 gauge and 22 gauge in the water phase. The relaxation of the surface tension, the volume relaxations of the bubbles, and the dependence of the surface tension on the bubble volume are as presented in Ref. [10]. As the bubble volume increases, the surface tension becomes higher and closer to the literature value, i.e. $72.0 \mathrm{mN} \mathrm{m}^{-1}$ at $25^{\circ} \mathrm{C}$. Fig. 3 shows the experimental data for 16 gauge and 22 gauge needles. The large, bold circle indicates the maximum bubble volume calculated theoretically. While the deviation of the surface tension increases as the bubble volume decreases for both the 16 gauge and the 22 gauge needles, the needle with the larger diameter does have a larger volume range in which the surface tension is $72.0 \pm 0.2 \mathrm{mN} \mathrm{m}^{-1}$ (see Fig. 10 also). For example, the value of the surface tension measured using a 16 gauge needle is higher than $71.8 \mathrm{mN} \mathrm{m}^{-1}$ and less than $72.2 \mathrm{mN} \mathrm{m}^{-1}$ when the bubble volume is larger than $11.5 \mathrm{~mm}^{3}$. The volume range for obtaining the $72.0 \pm 0.2 \mathrm{mN} \mathrm{m}^{-1}$

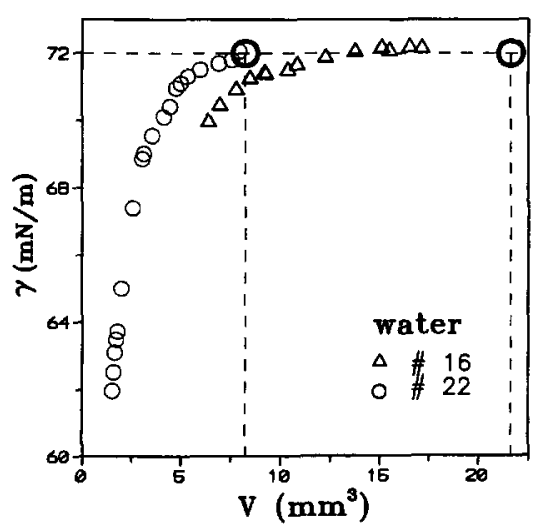

Fig. 3. Experimental values of the surface tension $\left(\mathrm{mN} \mathrm{m}^{-1}\right)$ of the air-water interface for bubbles of different sizes obtained by using 16 gauge $(\triangle)$ and 22 gauge $(O)$ needles at $25 \pm 0.02^{\circ} \mathrm{C}$. The large, bold circles denote the maximum bubble volumes. 
surface tension is more than $10 \mathrm{~mm}^{3}\left(V_{\max }=\right.$ $21.6 \mathrm{~mm}^{3}$ ). When a 22 gauge needle is used, the volume range for obtaining a tension of $\pm 0.2 \mathrm{mN}$ $\mathrm{m}^{-1}$ decrease to $0.7 \mathrm{~mm}^{3}$ (between 8.2 and $7.5 \mathrm{~mm}^{3}$ ). Note that the standard deviation for the best fit between several hundred edge coordinates of a bubble and the theoretical profile of the Young-Laplace equation only varies slightly for bubbles of different volumes, and the value of the deviation is in the same order as the error caused by the apparatus itself.

It has been shown in Ref. [10] that (i) the worst fit between the image data points and the theoretical curves always occurs at the region near the point of contact with the needle; (ii) the best fit curve is always perfectly fitted to the edge data points no matter how small the bubble and how large the deviation of the surface tension results; (iii) the largest deviation between the edge data points and the theoretical profile with the correct value of the surface tension is only around 1 pixel (approximately $10 \mu \mathrm{m}$ ) even for the smallest bubble; (iv) a 1 pixel deviation is almost the accuracy limit of the edge detection in the image digitization technique.

A similar tendency is also found in the glycerol system. Air bubbles with different volumes are also created on the tips of needles of gauges 16,22 , and 24 in the glycerol phase. The dependence of the measured surface tensions on the bubble volume is shown in Fig. 4. Note that a larger air bubble yields a surface tension closer to the correct value. A larger needle also has a larger volume range for obtaining a value of $62.9 \pm 0.2 \mathrm{mN} \mathrm{m}^{-1}$, as shown in Fig. 10 (see below). For example, the value of the surface tension measured using a 16 gauge needle is higher than $62.7 \mathrm{mN} \mathrm{m}^{-1}$ when the bubble volume is larger than $12.0 \mathrm{~mm}^{3}$. The volume range for obtaining a $62.9 \pm 0.2 \mathrm{mN} \mathrm{m}^{-1}$ surface tension is about $2.5 \mathrm{~mm}^{3}\left(V_{\max }=14.5 \mathrm{~mm}^{3}\right)$. When a 22 gauge needle is used, the volume range for obtaining an error within $\pm 0.2 \mathrm{mN} \mathrm{m}^{-1}$ is $1.2 \mathrm{~mm}^{3}$ (between 4.3 and $5.6 \mathrm{~mm}^{3}$ ). The reliable volume range decreases $0.7 \mathrm{~mm}^{3}$ (between 3.6 and $4.3 \mathrm{~mm}^{3}$ ) for a 24 gauge needle under the same conditions. A similar result is also obtained for the air/ethylene glycol system by using 22 and 24 gauge needles, as shown in Fig. 5.

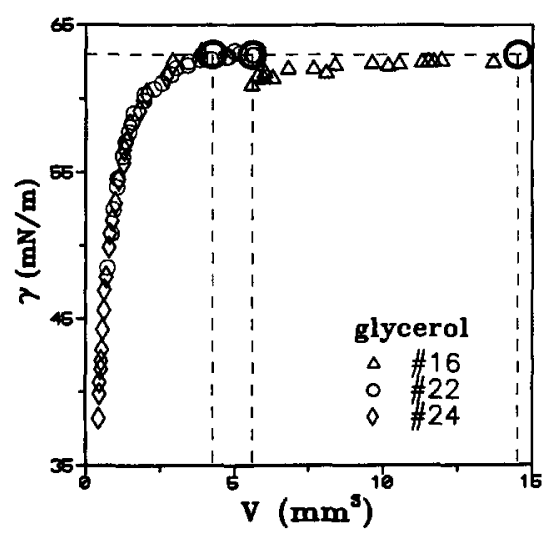

Fig. 4. Experimental values of the surface tension $\left(\mathrm{mN} \mathrm{m}^{-1}\right)$ of the air-glycerol interface for bubbles of different sizes obtained by using 16 gauge (4), 22 gauge $(O)$, and 24 gauge $(\diamond)$ needles at $25 \pm 0.02^{\circ} \mathrm{C}$. The large, bold circles denote the maximum bubble volumes.

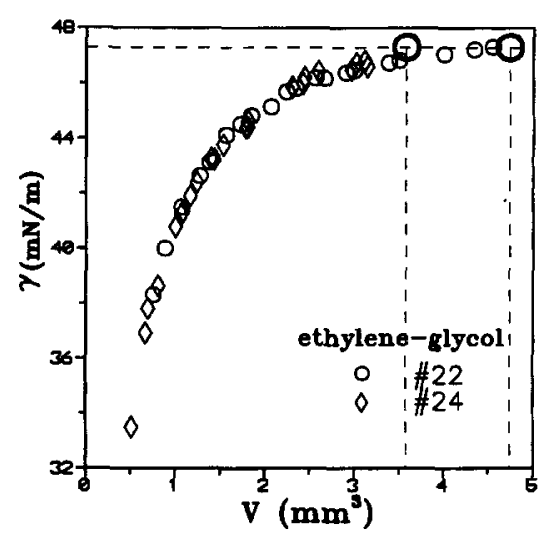

Fig. 5. Experimental values of the surface tension $\left(\mathrm{mN} \mathrm{m}^{-1}\right)$ of the air-ethylene glycol interface for bubbles of different sizes obtained by using 22 gauge $(O)$ and 24 gauge $(\diamond)$ needles at $25 \pm 0.02^{\circ} \mathrm{C}$. The large, bold circles denote the maximum bubble volumes.

Another two measurements for the air/benzaldehyde and air/toluene systems are also performed using a 22 gauge needle. Fig. 6 shows the measured surface tensions as a function of the pendant bubble volume for the 22 gauge needle. This indicates that all these five liquids show a similar behavior with respect to the surface tension measured using the pendant drop shape technique. Even for a smaller needle (24 gauge, shown in Fig. 7) or a larger needle (16 gauge, in Fig. 8), a similar relationship is also obtained. The only difference between Figs 


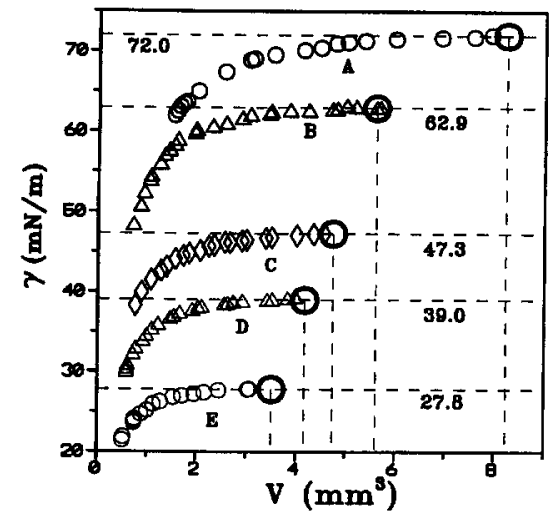

Fig. 6. Experimental values of the surface tension $\left(\mathrm{mN} \mathrm{m}^{-1}\right)$ of air-liquid interfaces for bubbles of different sizes obtained by using a 22 gauge needle at $25 \pm 0.02^{\circ} \mathrm{C}$ : curve $\mathrm{A}$, water; curve B, glycol; curve C, ethylene glycol; curve D, benzaldehyde; and curve $E$, toluene. The large, bold circles denote the maximum bubble volumes.

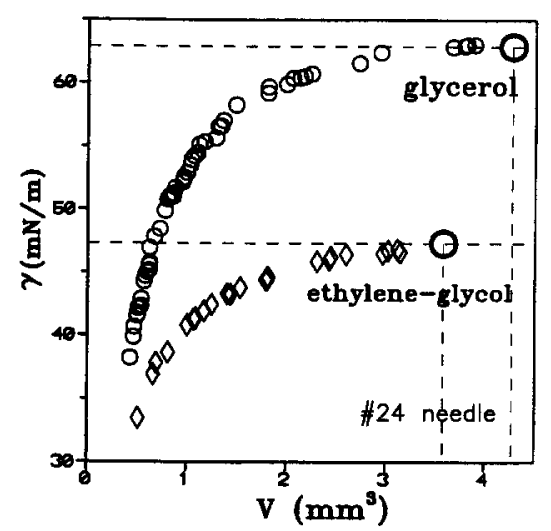

Fig. 7. Experimental values of the surface tension $\left(\mathrm{mN} \mathrm{m}^{-1}\right)$ of air-glycerol and air-ethylene glycol interfaces for bubbles of different sizes obtained by using a 24 gauge needle at $25 \pm 0.02^{\circ} \mathrm{C}$. The large, bold circles denote the maximum bubble volumes.

7,8 and 9 is that for a larger needle, there is a larger volume region capable of obtaining a correct value of the surface tension with an error of less than $\pm 0.2 \mathrm{mN} \mathrm{m}^{-1}$ (see Fig. 10 also). This is due to the fact that bubble profiles with a larger absolute value of $B$ deviate further from one another [10]. A larger needle yields a larger bubble, and a larger bubble has a larger value of $R_{0}$ (radius of curvature at the apex). Therefore, bubbles with larger volumes have larger absolute

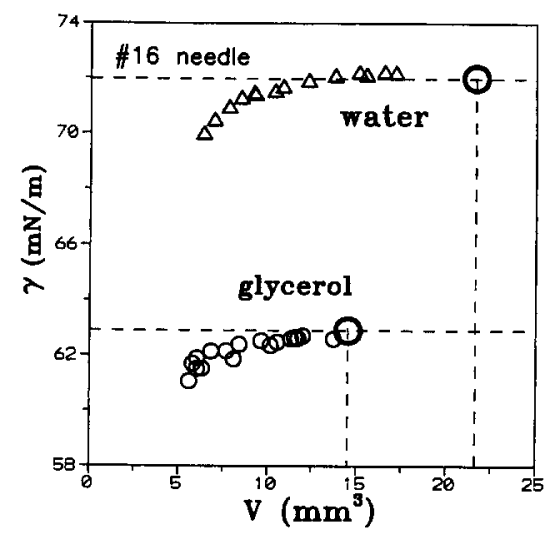

Fig. 8. Experimental values of the surface tension $\left(\mathrm{mN} \mathrm{m}^{-1}\right)$ of air-water and air-glycerol interfaces for bubbles of different sizes by using a 16 gauge needle at $25 \pm 0.02^{\circ} \mathrm{C}$. The large, bold circles denote the maximum bubble volumes.
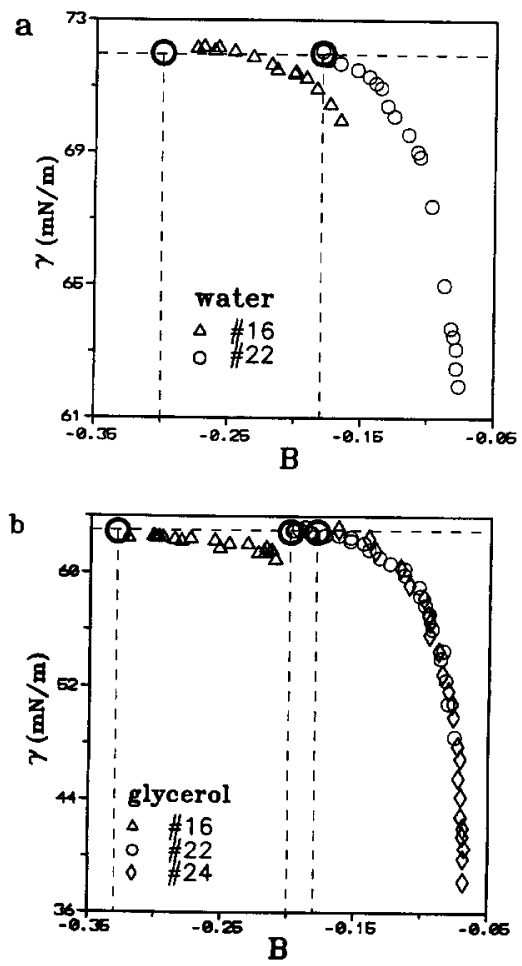

Fig. 9. Experimental values of the surface tensions $\left(\mathrm{mN} \mathrm{m}^{-1}\right)$ of air-water (a) and air-glycerol (b) interfaces for bubbles of different capillary constants corresponding to data in Fig. 3 and 5 . 
values of $B$. This dependence of the measured surface tension on $B$ for both water and glycerol is shown in Fig. 9.

\section{Discussion and conclusions}

In order to use these ideas successfully to predict the error of surface tension measurement by the pendant drop apparatus, we replot the data in Figs. 3-8. Figs. 10 and 11 show the bubble volume needed in order to obtain a surface tension with an error of less than 0.2 (shown by the squares) or
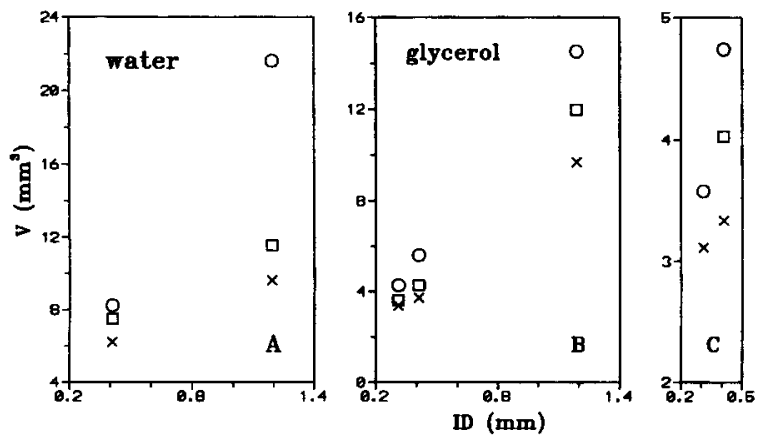

Fig. 10. Values of the bubble volume for obtaining a surface tension with an error of less than 0.2 or $0.5 \mathrm{mN} \mathrm{m}^{-1}$ as a function of the inner diameter of the needles used for the liquids water, glycerol, and ethylene glycol: $O$, maximum bubble volume; $\square, \pm 0.2 \mathrm{mN} \mathrm{m}^{-1} ; x, \pm 0.5 \mathrm{mN} \mathrm{m}^{-1}$. A, Water; B, glycerol; C, ethylene glycol.

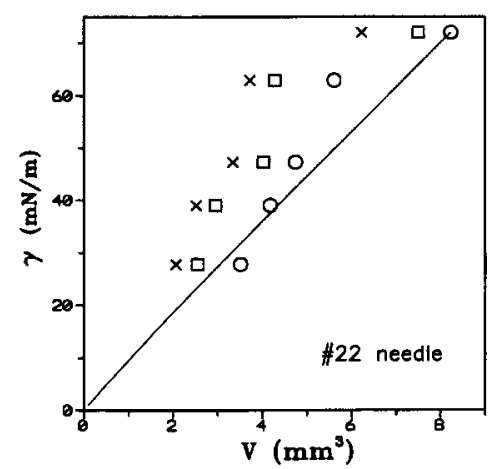

Fig. 11. Values of the bubble volume for obtaining a surface tension with an error of less than 0.2 or $0.5 \mathrm{mN} \mathrm{m}^{-1}$ as a function of surface tension measured using a 22 gauge needle: $O$, maximum bubble volume; $\square, \pm 0.2 \mathrm{mN} \mathrm{m}^{-1} ; \times$, $\pm 0.5 \mathrm{mN} \mathrm{m}^{-1}$.
$0.5 \mathrm{mN} \mathrm{m}^{-1}$ (shown by the crosses) from the drop shape for the five liquid systems mentioned above. The maximum bubble volume is also shown in Figs. 10 and 11 by the circles. Fig. 10 indicates clearly that a larger needle does have a larger volume range for obtaining an acceptable surface tension (with a measurement error of less than \pm 0.2 or $\pm 0.5 \mathrm{mN} \mathrm{m}^{-1}$ ). For example, the volumes for obtaining the surface tension of the air-glycerol interface within an error of $\pm 0.5 \mathrm{~m} \mathrm{~N} \mathrm{~m}^{-1}$ are 4.8 , 1.9 , and $0.9 \mathrm{~mm}^{3}$ for the 16,22 , and 24 gauge needles, respectively. The value for the air-water interface, for obtaining a tension within $0.5 \mathrm{mN}$ $\mathrm{m}^{-1}$, decreases from 12 to $2 \mathrm{~mm}^{3}$ for 16 and 22 gauge needles. Fig. 11 shows that the measurement error is a weak function of the surface tension of the liquids. The reliable volume range for obtaining a surface tension within an error of $\pm 0.5 \mathrm{mN} \mathrm{m}^{-1}$ is around $2 \mathrm{~mm}^{3}$ for all five liquid systems when a 22 gauge needle is used.

It would be helpful if there existed a universal relationship between the measurement errors of the pendant bubble technique and the bubble volumes. Fig. 12 shows a nearly universal relationship between the dimensionless surface tension measured, $\gamma^{*}=\gamma / \gamma_{\text {literature }}$, and a dimensionless group, $-B V^{*}$, where $B$ is the capillary constant

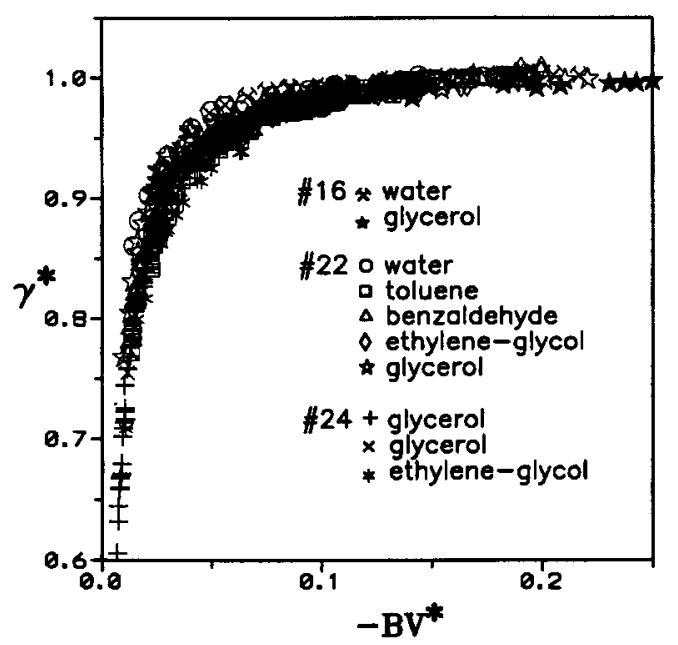

Fig. 12. Non-dimensional surface tension $\left(\gamma^{*}=\gamma / \gamma_{\text {literature }}\right)$ measured by the pendant bubble profile technique as a function of a non-dimensional combination parameter $B V^{*}\left(V^{*}=V / V_{\max }\right.$; $B$ is the capillary constant). 


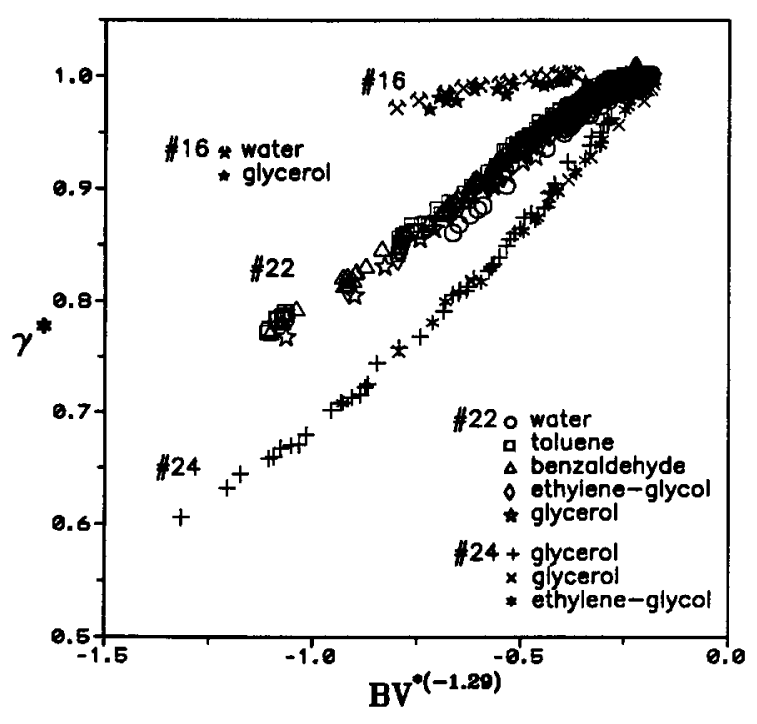

Fig. 13. Linear dependence of the non-dimensional surface tension $\left(\gamma^{*}=\gamma / \gamma_{\text {literature }}\right)$ measured by the pendant bubble profile technique on a non-dimensional combination parameter $B\left(V^{*}\right)^{-1.29}\left(V^{*}=V / V_{\max } ; B\right.$ is the capillary constant $)$.

and $V^{*}$ is the dimensionless bubble volume $\left(V^{*}=\right.$ $\left.V / V_{\max }\right)$. On the other hand, Fig. 13 shows that the dimensionless surface tension $\gamma^{*}$ is directly proportional to the dimensionless group $B\left(V^{*}\right)^{-1.29}$. The slope of this linear dependence varies with needle size. As one can see a larger needle gives a smaller slope. In other words, the dimensionless surface tension is a weaker function of $B\left(V^{*}\right)^{-1.29}$ for larger needles. More precisely, the bubble volume dependence of the surface tension resulting from the bubble profiles is weaker for a larger needle.

It should be pointed out that the universal relationship given in Figs. 12 and 13 is strictly valid for our system $(\times 1.5$ magnification, $480 \times 512$ resolution, and $10 \mu \mathrm{m}$ per pixel), schematically shown in Fig. 1. It is strongly believed that this systematic effect of the bubble volume on the surface tension measurements obtained by the drop profile technique exists in most of the drop shape systems. Therefore a careful examination, more precisely a calibration of the system, is necessary for accurate measurements. There have been several suggestions for improvements relating to the more accurate measurement of surface tensions
[10]. For example, the image should be enlarged by a higher magnification.

Garandet [13] analyzed the detachment conditions of a pendant drop and proposed an equation for calculating the maximum drop volume:

$2 \pi r_{0} \gamma=m g+\pi r_{0}^{2}\left(\frac{2}{R_{0}}-\rho g z_{0}\right)$

where $r_{0}$ is the radius of the needle, $m g$ is the weight of the liquid drop, and $z_{0}$ is the vertical distance between the apex and the bubble neck. In this equation, five unknowns $\Delta \rho, \gamma, r_{0}, z_{0}$ and $R_{0}$ are needed to calculate the maximum bubble volume. As a matter of fact, our method of theoretical calculation of the maximum bubble volume is essentially the same as that using Eq. (4). However, there are only three unknowns $\Delta \rho, \gamma$ and $r_{0}$, needed in our method.

In summary, the systematic effect of the bubble volume on surface tension measurements by using the pendant bubble shape technique on an airliquid interface has been investigated in this study. All five air/liquid systems used in this study show that the measured surface tension is bubble-size dependent. The deviation of the surface tension measured by the pendant bubble profile technique decreases as the bubble volume approaches the maximum bubble volume for all 16, 22, and 24 gauge needles. Besides, the needle with a larger diameter has a larger volume range for obtaining a correct surface tension (with a measurement error of less than 0.2 or $0.5 \mathrm{mN} \mathrm{m}^{-1}$ ). Bubbles with smaller absolute values of the capillary constant have larger measurement errors, in agreement with Lin et al. [10] due to the accuracy limit on the edge detection of a bubble image. A universal relationship for our system between the measured dimensionless surface tension $\gamma^{*}$, the capillary constant $B$, and the dimensionless bubble volume $V^{*}$ is also presented.

\section{Acknowledgements}

This work was supported in part by grants from the National Science Council, Taiwan (NSC 82-0402-E-011-199 and 84-2214-E002-004). 


\section{References}

[1] H.H.J. Girault, D.J. Schiffrin and B.D.V. Smith, J. Electroanal. Chem., 137 (1982) 207.

[2] Y. Rotenberg, L. Barouka and A.W. Neumann, J. Colloid Interface Sci., 93 (1983) 169.

[3] C. Huh and R.L. Reed, J. Colloid Interface Sci., 91 (1983) 472.

[4] A.W. Adamson, Physical Chemistry of Surfaces, 5th edn., Wiley, New York, 1990, Chapter 1.

[5] S.Y. Lin, K. McKeigue and C. Maldarelli, AIChE J., 36 (1990) 1785 .

[6] R. Miller, P. Joos and V.B. Fainerman, Adv. Colloid Interface Sci., 49 (1994) 249.
[7] C.H. Chang and E.I. Franses, Colloid Surfaces, 100 (1995) 1.

[8] J. Satherley, H.H.J. Girault and D.J. Schiffrin, J. Colloid Interface Sci., 136 (1990) 574.

[9] S.Y. Lin and H.F. Hwang, Langmuir, 10 (1994) 4703.

[10] S.Y. Lin, L.J. Chen, J.W. Xyu and W.J. Wang, Langmuir, 11 (1995) 4159

[11] P. Cheng, D. Li, L. Boruvka, Y. Rotenberg and A.W. Neumann, Colloids Surfaces, 43 (1990) 151.

[12] B. Carnahan, H.A. Luther and J.O. Wilkes, Applied Numerical Methods, Wiley, New York, 1969.

[13] J.P. Garandet, B. Vinet and P. Fros, J. Colloid Interface Sci., 165 (1994) 351. 